\title{
Robert Beale - ein englischer Diplomat mit europäischer Ausbildung
}

\section{Die frühen Jahre in Europa}

Robert Beale wurde ungefähr 1541 in London geboren, aber, wie der renommierte Frühneuzeithistoriker Patrick Collinson bemerkte, „it is the beginning of wisdom to understand that Beale was anything but a little Englander". ${ }^{1}$ Er war der Sohn eines Londoner Textilhändlers, der ebenfalls Robert mit Vornamen hieß. Vermutlich hatte seine Familie Verbindungen nach Suffolk (außerhalb von London); jedoch scheint der jüngere Robert nie Interesse an diesem Landstrich gehabt zu haben. Er wurde in den Midlands in Coventry ${ }^{2}$ in einer Elementarschule ausgebildet, die von dem Humanisten John Hales (1516?-1572) gegründet wurde. Beale verließ England während der Herrschaft von Queen Mary und floh ins Alte Reich, das in dieser Zeit für protestantische Exilanten aus England einen sicheren Schutzraum bot. Der Ort Coventry blieb Beale aber sein Leben lang lieb und teuer, denn er wurde später in den 1580er Jahren zum Beschützer für die Neffen und Freunde seines einstigen Lehrers John Hales, die in und um Coventry lebten. ${ }^{3}$ Wahrscheinlich waren es John Hales oder Beales Onkel, Sir Richard Morison (1510 - 1556), die Robert Beale dazu brachten, im Jahre 1553 oder 1554 aus England

1 Patrick Collinson: Servants and Citizens. Robert Beale and other Elizabethans, in: Historical Research 79/206 (2006), S. 488-511, hier S. 501f.; ders.: This England. Essays on the English Nation and Commonwealth in the Sixteenth Century. Manchester 2011, S. 109; die besten biographischen Informationen finden sich in der unveröffentlichten Dissertation von Mark Taviner: Robert Beale and the Elizabethan Polity. Diss. phil. University of St. Andrews 2000, und in meiner geplanten eigenen Biographie über Beale; Umrisse von Beales Biographie, mit einigen Fehlern, in: Gary M. Bell: Art. Beale, Robert, in: Oxford Dictionary of National Biography. Online-Version vom 28.05.2015. https://doi.org/10.1093/ref:odnb/1804 (abgerufen am 15.11.2018); J.C.H./P.W. Hasler: Art. Beale, Robert (1541-1601), of Barn Elms, Surr., Priors Marston, Warws. and of London, in: P.W. Hasler (Hrsg.), The History of Parliament. The House of Commons. Bd. 1. London 1981, S. 411- 414.

2 Collinson: Servants and Citizens, S. 501f.; ders.: This England, S. 109.

3 Siehe z. B. Beales Dokumente über St. Mary's, Coventry, in: British Library (künftig: BL), Additional MS 32100; Parishes: Priors Marston, in: Louis F. Salzman (Hrsg.), A History of the County of Warwick. Bd. 5: Kington Hundred. London 1949, S. 140 -141; vgl. Patrick Collinson: The Elizabethan Puritan Movement. London/New York 1982, S. 414f.; über Beales eigene Gedanken zu Coventry im späteren Leben siehe Beale an Whitgift, 22.08.1590, BL, Additional MS 48039, fol. $74 \mathrm{r}$. 
zu fliehen - zu dieser Zeit war der junge Mann gerade erst etwa zwölf oder dreizehn Jahre alt. Hales spielte weiterhin eine entscheidende, beinahe väterliche Rolle in Beales Leben: Während der 1550er und 1560er Jahre brachte er ihn in Kontakt mit bedeutenden politischen und religiösen Persönlichkeiten wie dem Großschatzmeister Polens, Spytek Jordan, mit dem religiösen Flüchtling Jan Łaski und mit dem lutherische Theologen Georg Maior. ${ }^{4}$ Über den Aufenthalt von Beales Geschwistern oder seiner Mutter ist nichts bekannt. Sein Vater war 1548 gestorben, John Hales kann daher als Beales Ersatzvater gelten. ${ }^{5}$

Beale lebte in den 1550er Jahren zunächst in Straßburg bei seinem Onkel, Sir Richard Morison, und John Abell, einem Londoner Kaufmannsbanker. ${ }^{6}$ Er war dort Teil einer kleinen, aber geschützten englischen Gemeinschaft protestantischer Exilanten, aus der später viele prominente Kirchenmänner und Regierungsbeamte des elisabethanischen Englands hervorgehen sollten. ${ }^{7}$ Beales Zeit in der Freien Reichstadt Straßburg war absolut entscheidend für seine spätere Entwicklung. Er besuchte dort die Vorlesungen von Peter Martyr und Johann(es) Sturm, aber sein Lehrmeister war John Aylmer, der einstige Hauslehrer von Lady Jane Grey (1537-1554) und zukünftige Bischof von London, der offenbar nicht zögerte, den junge Beale mit einer Rute zu schlagen. ${ }^{8}$ Während seiner Zeit in Straßburg zählte Beale $\mathrm{zu}$ einer besonders fortschrittlichen protestantischen Gruppe, der mehrere Jugendliche angehörten. ${ }^{9}$

Im Jahre 1556 oder 1557 zog er mit Aylmer nach Zürich und blieb dort etwa ein Jahr. Sein Mentor Aylmer kümmerte sich nicht mehr um ihn, sondern überließ den jungen Beale sich selbst. ${ }^{10}$ Zürich war die Heimat einer anderen Gemeinschaft

4 Aberdeen University Library (künftig: AUL), MS 1009/1, Artikel 11, 12; Taviner, Robert Beale, S. 49.

5 Vgl. Robert Beales (der Ältere) letzter Wille und Testament, The National Archives (künftig: TNA), PROB 11/32/171.

6 Tracey A. Sowerby: Renaissance and Reform in Tudor England. The Careers of Sir Richard Morison c.1513-1556. Oxford 2010, S. 223-232.

7 Über die englischen Exilanten vgl. Christina Hallowell Garrett: The Marian Exiles. A Study in the Origins of Elizabethan Puritanism. Cambridge 1938.

8 The answer of Robert Beale concerninge such thinges as have passed between the L. Archbishopp of Canterburye and him, 01.07.1584, BL, Additional MS 48039, fol. 48r-v; über Martyr und Sturm siehe R. Gerald Hobbs: Strasbourg. Vermigli and the Senior School, in: Torrance Kirby/Frank A. James II/Emidio Campi (Hrsg.), A Companion to Peter Martyr Vermigli. Leiden 2009, S. 35-69; Lewis W. Spitz/Barbara Sher Tinsley: Johann Sturm on Education: The Reformation and Humanist Learning. St. Louis, Mo. 1995.

9 So ist z.B. belegt, dass Aylmer einen weiteren jungen Mann namens Thomas Dannett als Schüler hatte; vgl. Danett an Burghley, 01.05.1595, Hatfield House, Cecil Papers (künftig: CP), 32/ 14.

10 BL, Additional MS 48039, fol. 48r. 
englischer protestantischer Exilanten, die in ihren theologischen Lehrmeinungen, religiösen Doktrinen und in ihrer konfessionellen Disziplin wesentlich strenger und kompromissloser waren. ${ }^{11}$ Aylmer passte wegen seiner eigenen theologischen Überzeugungen gut zu dieser Züricher Gruppe. Beale scheint dagegen die Zeit in Zürich nicht sonderlich genossen zu haben. Später im Leben ließ er nie viel über den Ort oder die Leute verlauten, die er dort traf.

Ein nächster Hinweis auf seinen darauffolgenden Aufenthaltsort und seine Aktivitäten stammt aus Beales eigener Erinnerung - beiläufig erwähnt in einem Buch über religiöse Kontroversen, das er 1584 für den Erzbischof von Canterbury geschrieben hat und das als eine Verteidigung von Beales intellektuellen und religiösen Positionen gelesen werden kann. Beale bemerkte darin, dass sein Studium des Zivilrechts 26 Jahre zurückliege. Es lässt sich somit auf das Jahr 1558 datieren. $^{12}$

Als Elisabeth I. im November 1558 auf den Thron kam, eilten die meisten protestantischen Männer und Frauen aus dem deutschen und schweizerischen Exil zurück in ihr Heimatland, Beale jedoch nicht. Als sein Lehrmeister Aylmer Zürich Anfang 1558 verließ, um an die Universität der Ernestiner in Jena zu gehen, zog Beale wahrscheinlich von Zürich nach Heidelberg. ${ }^{13}$ Angesichts der wenigen Quellen ist es zwar sehr kompliziert, Beale zu dieser Zeit in Heidelberg zu verorten. Jedoch scheint seine spätere Korrespondenz mit Franz Balduin (Balduinus, eigentl. François Baudouin, 1520 - 1573), Professor für Zivilrecht an der Universität Heidelberg, in diese Richtung zu weisen. ${ }^{14}$ Beale und Balduin verband ein sehr ähnliches Interesse und Verständnis für Recht und Geschichte, das Beale möglicherweis erst von Balduin erlernte.

Balduin lehrte an der Universität von Straßburg zu der Zeit, als auch Beale dort in den Jahren 1555 bis 1556 studierte. ${ }^{15}$ Nach seinem Wechsel an die Uni-

11 Über die Engländer in Zürich siehe ihre Korrespondenzen in Hastings Robinson (Hrsg.): The Zurich Letters. Cambridge 1842, 1845, 1846; ders. (Hrsg.): Original Letters Relative to the English Reformation. Cambridge 1846, 1847.

12 Vgl. Beale an Whitgift, 07.05.1584, BL, Additional MS 48039, fol. 42r-45v.

13 Brett Usher: Art. Aymer, John, in: Oxford Dictionary of National Biography. Online-Version vom 03.01.2008. https://doi.org/10.1093/ref:odnb/935 (abgerufen am 15.11.2018); Johann Friedrich II. an John Hales, 01.01.1559, BL, Additional MS 15943, fol. 1r-2r; Taviner dachte, dass Beale vielleicht an die Universität von Padua gegangen wäre, aber diesbezüglich gibt es keine direkten Beweise (s.u.).

14 AUL, MS 1009/2, Artikel 35-38.

15 Alexander Russell: The Colloquy of Poissy, François Baudouin and English Protestant Identity, 1561-1563, in: The Journal of Ecclesiastical History 65/3 (2014), S. 551-579, hier S. 569; über Balduin im Allgemeinen siehe: Michael Erbe: François Bauduin (1520 -1573). Biographie eines Humanisten. Gütersloh 1978; Mario Turchetti: Concordia o Tolleranza? François Bauduin (1520 - 
versität Heidelberg stieg Balduin zwischen 1556 und $1561 \mathrm{zu}$ einem hoch angesehenen Professor für Zivilrecht auf und entwickelte in Auseinandersetzung mit den lutherischen Ideen eine besondere Sicht auf die Geschichte, sei es auf einzelne Ereignisse oder auf ganze Epochen. Seine Freundschaft mit Philipp Melanchthon scheint dabei nicht unerheblich gewesen zu sein. Folgt man der modernen Forschung, dann sind in Balduins Heidelberger Werken eindeutig Melanchthons Ideen zur Geschichte aus lutherischem Geist zu erkennen. Das ist bemerkenswert, da Balduin zuvor eine längere Zeit dem Calvinismus, später dem Katholizismus zugeneigt war. ${ }^{16}$ Balduin griff Melanchthons Ideen auf, dachte sie weiter und trat in seinen Vorlesungen für eine „new wave of legal Humanism“ ein. Das tat er zuerst in Straßburg 1555 und dann intensiver und ausführlicher in Heidelberg 1559; im Jahre 1561 wurden seine Ideen schließlich veröffentlicht. ${ }^{17}$ Balduins Kombination von Recht, Geschichte und Protestantismus in Heidelberg lieferte wichtige (methodische) Argumente für die Etablierung der Geschichte als Wissenschaft in Deutschland und Frankreich. ${ }^{18}$ Allerdings hatte er auch Einfluss auf Engländer, zumindest knüpfte er auch Kontakte zu Beales englischen Landsleuten.

Zur selben Zeit studierte nämlich ein weiterer Engländer in Heidelberg, Henry Killigrew (1525/28 -1603). Er hatte sich 1558 formell an der Universität immatrikuliert und war durch einen gemeinsamen Kontakt in Straßburg - Sir Anthony Cooke (1505/6 -1576) - mit Beale verbunden. Killigrew und Beale wurden später in der Regierung von Königin Elisabeth I. enge Kollegen. Killigrew, Cooke und Balduin scheinen in Heidelberg Freundschaft geschlossen zu haben, und so ist es denkbar, dass Beale dem Zivilrechtsprofessor Balduin auf einer persönlichen Ebene begegnete, nicht nur im akademischen Milieu. ${ }^{19}$ Balduins Freundschaft mit

1573) e i „Moyenneurs“. Milano 1984; Donald Kelley: Historia Integra. François Baudouin and his Conception of History, in: Journal of the History of Ideas 25/1 (1964), S. 35-57; zu Balduins Zeit in Heidelberg siehe Universitätsarchiv Heidelberg, RA 659, 660, 662.

16 Z. B. Lars Boje Mortensen: François Bauduin’s „De Institutione Historiæ“ (1561). A Primary Text behind Anders Sørensen Vedel's „De Scribenda Historia Danica“ (1578), in: Symbolae Osloenses. Norwegian Journal of Greek and Latin Studies 73 (1998), S. 188-200; vgl. Barbara Pitkin: Calvin's Mosaic Harmony. Biblical Exegesis and Early Modern Legal History, in: The Sixteenth Century Journal 41/2 (2010), S. 441- 466.

17 Pitkin, Calvin's Mosaic Harmony, S. 458, 462.

18 Wie Donald Kelley betont, bot Balduin ,the first serious attempt in France to formulate a definition of history - indeed, to promote history from an art to a science by organizing it in a methodical way“; vgl. Donald Kelley: Foundations of Modern Historical Scholarship: Language, Law, and History in the French Renaissance. New York 1970, S. 116; vgl. ders., Historia Integra. 19 Gustav Toepke (Hrsg.): Die Matrikel der Universität Heidelberg von 1386 bis 1662. Bd. 2. Heidelberg 1886, S. 16 (23.11.1558); Russell, The Colloquy of Poissy, S. 568; Balduin an Cecil, 15.03. 
Melanchthon und einem anderen gleichgesinnten Philippisten, Hubert Languet (1518-1581), begann 1557 auf dem Wormser Kolloquium. Um dieselbe Zeit schloss auch Beale eine bleibende Freundschaft mit Languet, lernte Melanchthon selbst aber nie kennen. Weitere mögliche personelle Verbindungen zwischen Balduin und Beale sind der Jurist und spätere pfälzische Geheimrat Christoph Ehem (1528-1592) und der englische Agent in Deutschland Christoph Mundt (1496/71572). ${ }^{20}$ Der Einfluss der melanchthonschen Umgebung Heidelbergs in den späten 1550er Jahren auf Beales intellektuelle Entwicklung ist zwar nur schwer zu bestimmen. Auffällig ist aber, dass Beale die gleichen Ideen über Geschichte, Recht und Religion vertrat wie Balduin und dass beide denselben Personenkreis kannten. Die Verbindung zwischen den beiden Männern wurde erst in den 1560er Jahren fester, sie scheint aber schon 1558 in Heidelberg ihren Anfang genommen $\mathrm{zu}$ haben.

Beales Aktivitäten in Heidelberg und seine Vertrautheit mit der Stadt in den 1560er Jahren unterstützen die These, dass er schon früher dort war. Unklar ist gleichwohl, was er im Alter von 17 oder 18 Jahren dort machte, ob er Zivilrecht studierte oder versuchte, von Zürich nach England zurückzukehren. In jedem Falle scheint er die Unterstützung anderer Engländer mit Verbindung nach Straßburg gehabt zu haben. Der junge Beale hatte einige einflussreiche Unterstützer wie Richard Morison und John Aylmer, ${ }^{21}$ dennoch reiste und lebte er zum größten Teil allein. Es gibt nur wenige spekulative Anhaltspunkte, dass Beale in den 1550er oder 1560er Jahren nach Italien reiste, um Rechtswissenschaften an der Universität von Padua zu studieren. ${ }^{22}$ Wesentlich wahrscheinlicher ist es, dass sein Interesse an Zivilrecht und Geschichte vor allem von Balduin in Heidelberg gefördert und später in Paris fortgeführt wurde (wo Balduin auch für einen Großteil der 1560er Jahre residierte). Es ist bemerkenswert und wohl mehr als ein Zufall, dass auch Balduin zunehmend an englischen Angelegenheiten interessiert war, direkt an Minister der englischen Regierung und Vertreter der Kirche schrieb und sich persönlich mit dem englischen Agenten in Deutschland, Christoph Mundt, austauschte. ${ }^{23}$ Noch wichtiger aber ist, dass Balduin am Anfang von Elisabeths I. Regierungszeit einen Traktat unmittelbar für die Königin schrieb - über die englische Thronfolge sowie die Gleichwertigkeit und Legitimität ihres Herr-

1559, TNA, SP 70/3, fol. 52r-53v; zum religiösen Charakter der Universität Heidelberg zu dieser Zeit siehe Charles D. Gunnoe, Jr.: Thomas Erastus and the Palatinate. A Renaissance Physician in the Second Reformation. Leiden 2011, S. 56-62.

$20 \mathrm{Zu}$ Balduins Kontakten sieh Erbe, François Bauduin; Turchetti, Concordia o Tolleranza?

21 Vgl. oben.

22 Vgl. z.B. Taviner, Robert Beale, S. 54.

23 Balduin an Cecil, 15.03.1559, TNA, SP 70/3, fol. 52r-53v. 
schaftsanspruches. ${ }^{24}$ Beale schrieb zwei Jahre später (1563) ebenfalls zwei Abhandlungen über die königliche Nachfolge und die Legitimität des Anspruchs auf den englischen Thron, wenn auch von einer anderen Thronanwärterin. ${ }^{25}$ Bemerkenswert ist, dass Beale darin den vorgezeichneten Linien von Balduins Argumentation folgte.

Beales Ausbildung stand zweifellos unter dem Einfluss von Engländern, die dem Protestantismus in England in den 1540er und frühen 1550er Jahren den Weg bereitet hatten. Dennoch hatten seine Erfahrungen und Kontakte in lutherischen und reformierten Territorien des Alten Reiches während der 1550er Jahre wahrscheinlich einen größeren Einfluss auf die Entwicklung seiner Ideen in Bezug auf Religion und Politik im Allgemeinen und auf die englischen Beziehungen zu Protestanten in Deutschland im Besonderen. Es ist anzunehmen, dass sich Beale sein Wissen für den späteren Diplomatenberuf, insbesondere sein außenpolitisches Expertenwissen, in sehr jungen Jahren durch direkte Erfahrung und Austausch angeeignet hat.

Letztlich ist es aber schwierig herauszufinden, wo sich Beale in den 1550er Jahren aufhielt. Er war zu dieser Zeit ein Teenager ohne Familie, der in etlichen fremden Ländern unterwegs war. Er hatte nur die lose Unterstützung einer Gruppe von Exilanten, die versuchten, nirgendwo aufzufallen und sich aus Schwierigkeiten herauszuhalten. Beale selbst sagte später, dass er „took grat pains in travelling in divers countries on foot for lack of other abilities“. ${ }^{26}$ Wegen seines jungen Alters sind von ihm keine Steuerdokumente oder anderen offiziellen Dokumente in Straßburg, Zürich oder Heidelberg erhalten geblieben. Der große Frankfurter Verleger André Wechel notierte in den 1570er Jahren, dass Beale „fast alle Schulen Deutschlands, Frankreichs und Italiens besucht“ habe, jedoch lassen sich an den Universitäten von Paris oder Padua keine Immatrikulationsakten finden - es gibt somit keine Belege für gerade jene zwei Orte, die von der älteren historischen Forschung am ehesten mit Beales Zivilrechtslehre in Verbindung gebracht wurden. ${ }^{27}$ Obwohl sich Beales Name leider auch nicht in den Matrikeln der Universität Heidelberg findet, verweisen die übrigen späteren Quellen und personellen Verbindungen dennoch stark auf einen Aufenthalt in Heidelberg.

Mit großer Sicherheit lässt sich dagegen Beales Aufenthaltsort am 17. Mai 1560 bestimmen, weil er sich an diesem Tag formell an der Universität von Wittenberg

24 BL, Additional MS 48114, fol. 54r-68v.

25 Dazu ausführlicher im zweiten Teil des Aufsatzes.

26 Beale an Burghley, 1591, BL, Lansdowne MS 68/107, fol. 238r-v.

27 André Wechel: Rerum Hispanicarvm Scriptores Aliqvot, quorum nomina versa pagina indicabit. Ex Bibliotheca clarißimi viri Dn. Roberti Beli Angli. Bd. 1. Frankfurt 1579, fol. Iijr; Taviner, Robert Beale, S. 54. 
immatrikulierte. ${ }^{28}$ Er traf dort auf die meisten diplomatischen und politischen Vertreter der melanchthonschen oder reformierten Intellektuellen und intensivierte überdies seine Freundschaft mit Hubert Languet. ${ }^{29}$ Es gibt Hinweise darauf, dass sich Beale und Languet möglicherweise schon in Heidelberg getroffen haben. Mit Sicherheit kamen sie in Wittenberg zusammen. Sucht man in Europa nach einem verwandten Geist, der Beale in diplomatischer Hinsicht gleichkam, dann findet man ihn in der Person Hubert Languets. Dessen Verbindungen zu Melanchthon waren tief, sein Bekenntnis zum Protestantismus war international, und sein politischer Scharfsinn war zuspitzend und treffend.

War Beale seit Mitte der 1550er Jahren zunächst im westlichen Deutschland und in der Schweiz unterwegs, wurde er ab 1560 immer mehr mit dem östlichen Deutschland, insbesondere mit Sachsen vertraut. Die Entscheidung, sich in Wittenberg einzuschreiben, war bedeutsam. Es ist anzunehmen, dass Beale schon vor 1560 die theologischen und politischen Positionen des unnachgiebigen Lutheraners Matthias Flacius (Illyricus) bekannt waren, denn Flacius hatte großen Einfluss auf Beales früheren Tutor John Aylmer an der Universität Jena. ${ }^{30} \mathrm{Zu}$ diesem Zeitpunkt (1560) hatte sich Beale allerdings von harten, unnachgiebigen religiösen Positionen bereits entfernt, wenn er überhaupt je ein Hardliner gewesen ist; zudem war er mittlerweile durch eine Heidelberger Umgebung geformt und geprägt, zu der mehr theologisch gemäßigtere - und politisch geschicktere Akademiker und Diplomaten gehörten, als dies in Jena der Fall war. ${ }^{31}$ Ebenfalls aussagekräftig, aber noch nicht schlüssig zu erklären, ist Beales Besitz eines Briefes von Georg Maior (1502-1574) über Beales Ersatzvater, John Hales. ${ }^{32}$ Im Jahr 1560 war Georg Maior in Wittenberg, und vielleicht war er auch der Grund, warum Beale dorthin ging; Maior wurde später wie Languet $\mathrm{zu}$ einer der Schlüsselfiguren unter den Philippisten. ${ }^{33}$

Es spricht deutlich für Beales frühe und spätere Überzeugung, dass er zu den Philippisten in Wittenberg und nicht zum Gnesio-Lutherischen Kreis in Jena ging.

28 Karl Eduard Förstemann u.a. (Hrsg.): Album Academiae Vitebergensis. Ältere Reihe in 3 Bdn. 1502-1602. Bd. 2. Leipzig/Halle 1894, S. 4.

29 Über Languet, Melanchthon und Beale siehe Béatrice Nicollier-de Weck: Hubert Languet (1518-1581). Un Réseau Politique International de Melanchthon à Guilaume d’Orange. Genf 1995, insbes. S. 226f.; Taviner, Robert Beale, S. 64-66 und passim.

30 Vgl. BL, Additional MS 48039, fol. 55r.

$31 \mathrm{Vgl}$. Gunnoe, Thomas Erastus and the Palatinate.

32 AUL, MS 1009/1, Artikel 12.

33 Über Maior siehe Robert Kolb: Georg Major as Controversialist. Polemics in the Late Reformation, in: Church History 45/4 (1976), S. 455-468; Heinz Scheible: Art. Major, Georg, in: Gerhard Müller u. a. (Hrsg.), Theologische Realenzyklopädie (TRE). Bd. 21. Berlin/New York 1991, S. 725 730. 
Er mag die Strenge und Disziplin von Zürich nicht gemocht haben; oder er bevorzugte das politisch aktive Luthertum von Straßburg und Heidelberg. Wittenberg scheint er jedoch aus gutem Grund gewählt zu haben - zumindest lässt sich diese Entscheidung mit seinem moderaten Luthertum und seiner (späteren) politischen und diplomatischen Flexibilität gut begründen.

\section{Beales Schritte in die Welt der Diplomatie}

Da Beales Leben in den frühen 1560er Jahren prinzipiell schwer in den Quellen greifbar ist, bietet es sich an, seine 1563 angefertigte Rechtfertigungsschrift für John Hales im Namen von Edward Seymour, Graf von Hertford (1539?-1621), näher zu betrachten. Darin ging es um die Heirat des Grafen mit Catherine Grey, Schwester der Neun-Tage-Königin Lady Jane Grey, und um die Legitimität ihres Anspruchs auf die englische Thronfolge für den Fall, dass Elisabeth I. kinderlos sterben sollte. ${ }^{34}$ Damit war Beale zu einem sehr frühen Zeitpunkt in seinem Leben unmittelbar in Fragen um die Monarchie involviert - und das, obwohl er der königlichen Führungsriege noch relativ - wenn nicht ganz und gar - unbekannt war. Für einen politischen Außenseiter hätte diese Position sehr gefährlich werden können. Ziemlich sicher war es Hales gewesen, der Hertford Beale vorgeschlagen hatte - wegen Beales Interesse und seiner Kontakte zum Zivilrecht und wegen Balduins früherer Abhandlung über die königliche Thronfolge. Beale reaktivierte tatsächlich seine internationalen Kontakte und konsultierte nachweislich Juristen in Marburg, Köln, Speyer und (später) Paris mit Fragen über Hertfords Ehe und Greys Anspruch. ${ }^{35}$ Er schrieb über diese Frage seinen eigenen „lardge discourse“, folgte dabei Balduins Argumentationslinien und bestätigte sowohl die Legitimität der Seymour-Grey-Ehe als auch die Legitimität von Greys Anspruch auf den englischen Thron. Zudem schrieb Beale einen zweiten Traktat über die Legitimität der Ehe zwischen Charles Brandon, Herzog von Suffolk, und Frances Grey (Catherines Mutter), um die Richtigkeit seiner Behauptung weiter zu untermauern. ${ }^{36}$

Beale bewies in beiden Traktaten detailliertes juristisches Wissen und zeigte, dass er bereits mit 22 Jahren ein bedeutender Experte im kanonischen Recht wie auch im Zivilrecht war und zudem hervorragende Kontakte zur Rechtswissen-

34 Vgl. zu dieser Frage Mortimer Levine: The Early Elizabethan Succession Question 1558-1568. Stanford, Calif. 1966, S. 62-85.

35 Cambridge University Library, MS Ii.v.3.

36 Vgl. Cambridge University Library, MS Dd.iii.85, Artikel 18. 


\section{Species Farfi}

Sempromins. N. Nobifis, sin iuris existens, amabout Sezam

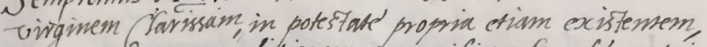
ate post fongam ambitionem, eam sibi tandem de' matri = monio ronsentive fait: sed clane ufu, ania timebat Dim

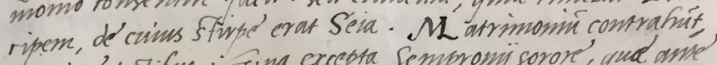

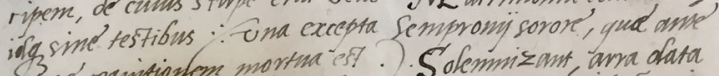
causa' roginisionfem mortana ies? Sofermizant, arra data ynoratw. I me protem ex ca mafenfam gignist, ac peregre

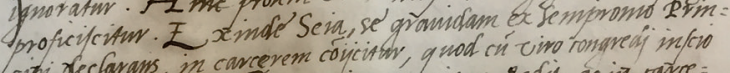

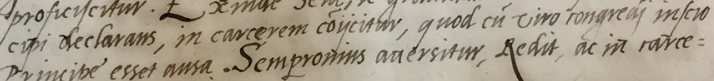
rem etian detrubliow

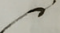

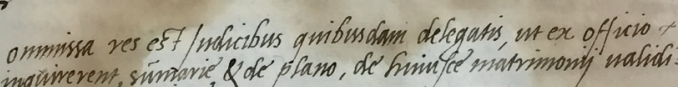

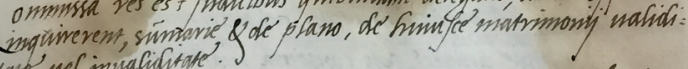

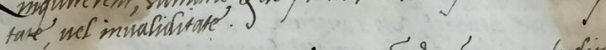

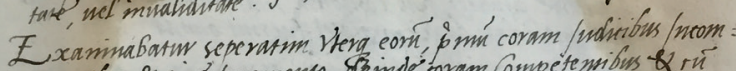
petensibus, \& sine /mramento, seinde foram Competemsibus of ri

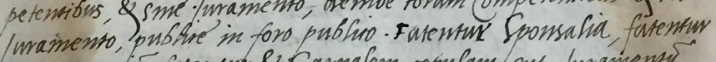

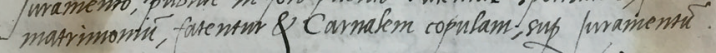

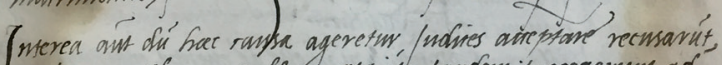

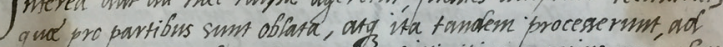
Sententian quandam, vt norowinn diffinitinash, evius exiénglow

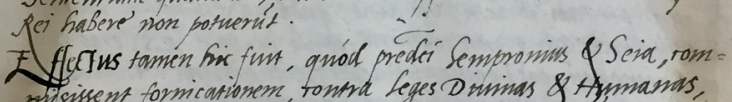
missisent formicationem, tontra leges D ininas \& Aymanas,

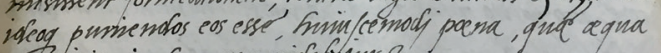
reguisita in foc casn misebitior?

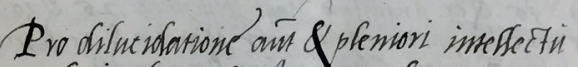 finims hy potheseos, deto ad sequentes.} quesfiones, plesissime, a Carisso ono Doctore respontion

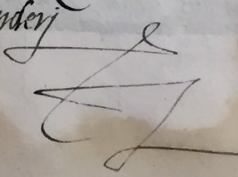

Abb. 4: Beales Traktat zum Abschluss der Hertford-Grey-Ehe (1563)

schaft pflegte - auch zu Juristen am deutschen Reichskammergericht. ${ }^{37}$ Wegen der politischen Spannungen, die Jane Greys Anspruch verursachte, erhielten Hales und andere ernste Drohungen und Tadel, aber nicht Beale - wahrscheinlich

37 Cambridge University Library, MS Ii.v.3, Artikel 3, 5. 
aufgrund seiner Jugend und seiner entfernteren Stellung. Dennoch lässt sich mit Fug und Recht behaupten, dass er spätestens seit 1563 in das Sichtfeld von William Cecil (1521-1598), dem engsten Berater und Hauptsekretär Königin Elisabeths, geriet. ${ }^{38}$ Cecil wurde später über lange Jahre Beales beständigster Unterstützer in der englischen Regierung und förderte dessen Karrierestart in der Außenpolitik.

Beale schickte seine Traktate sowie die rechtlichen Einschätzungen der deutschen Juristen zu Hales nach England. Ob Beale selbst in einer Mission oder mit einem spezifischen Auftrag nach England zurückgekehrt war, lässt sich schwer sagen. Er könnte als Kurier für Briefe zwischen London, Deutschland und Frankreich agiert haben. Letztlich lässt sich aber kaum belegen, wo sich Beale während dieser Bildungsjahre aufhielt. Als gesichert kann dagegen sein Aufenthalt in Paris gelten, wo er seit 1564 lebte. ${ }^{39}$ Beales Zeit und seine informellen Positionen in Paris während der mittleren und späten 1560er Jahre waren in vielerlei Hinsicht das Produkt und der Höhepunkt seiner zuvor erworbenen Erfahrungen. Denn obwohl sein Wissen und seine angehende Expertise in reformierten und lutherischen Angelegenheiten hauptsächlich deutsche und schweizerische Länder betrafen, war Paris der wichtigste Ort für die englische Diplomatie und enorm wichtig für das Sammeln von Nachrichten auf dem europäischen Festland. Wollte Beale sich einen Namen machen und die Achtung und Aufmerksamkeit potentieller Förderer und Unterstützer in der englischen Regierung auf sich ziehen, dann war Paris der Ort, wo er sein musste.

Während seiner Zeit in Paris diente Beale als enger Mitarbeiter und Kurier für die folgenden englischen Botschafter: Sir Thomas Smith (1513-1577), Sir Thomas Hoby (1530-1566), Hugh Fitzwilliam (ca. 1534-ca. 1576) und Sir Henry Norris (ca. 1525-1601). ${ }^{40}$ Es ist anzunehmen, dass Beale weiterhin Kontakt zu Balduin hatte, dem Professor für Zivilrecht, der 1561 als moderate Stimme bei den Religionsgesprächen im „Kolloquium von Poissy“ agiert hatte und danach nach Paris umzogen war. Vier Briefe von Balduin an Beale sind an der Universität von Aberdeen erhalten. Obwohl Balduin diese Briefe erst um 1570 in Paris und Angers schrieb, sind sie überaus wertvoll, da sie auf die frühere Korrespondenz und ihre persönliche Beziehung Bezug nehmen. ${ }^{41}$ In Paris scheint sich Beale auch mit dem oben genannt Verleger André Wechel angefreundet zu haben, der später eine

38 Viele Dokumente zu diesem Rechtsfall befinden sich im persönlichen Archiv von Cecil: CP $154 / 60-65$.

39 Beale an Burghley, 15.04.1578, BL, Lansdowne MS 27/32, fol. 63r.

40 Vgl. dazu auch Taviner, allerdings ohne die Jahre 1564/65: Taviner, Robert Beale, S. 70-103. 41 AUL, MS 1009/2, Artikel 35-38. 
große Sammlung spanischer Bücher aus Beales Bibliothek herausgab. ${ }^{42}$ Mitte der 1560er Jahre traf Beale in Paris auch wieder auf seinen Wittenberger Kommilitonen Hubert Languet, der während jener Jahre ebenfalls lange Zeit in Paris tätig war, nämlich als Botschafter des Kurfürsten von Sachsen. ${ }^{43}$

In Paris war Beale nun mittlerweile etwa 24 Jahre alt: Er war weit gereist, hatte in verschiedenen konfessionellen Umfeldern studiert (wenn auch zumeist informell) und begonnen, das diplomatische Handwerk und die Praxis der Diplomatie in der englischen Botschaft in Paris zu erlernen. Seine Auffassung von der Funktionsweise des Rechts im historischen Kontext war maßgeblich von Balduin geprägt worden, sein Verständnis für das Wechselspiel zwischen Politik und Religion verdankte er seinen Reisen und seiner Freundschaft mit Languet. Seine englischen Zeitgenossen gingen davon aus, dass er in erster Linie von Sir Thomas Smith lernte und von diesem beraten wurde. ${ }^{44}$

\section{Europäisches Wissen als Befähigung zum Diplomatendienst}

Beale trat vor 1566 in den Dienst von William Cecil ein, dem Hauptsekretär von Elisabeth I. Das geht aus einem überaus informativen Brief von Beale an Cecil über den englischen Botschafter in Paris hervor. ${ }^{45}$ Dieser Brief ist sehr aufschlussreich, weil er viel über Beales frühes Verständnis für die Feinheiten der religiösen und politischen Diplomatie aussagt.

Die sprachlichen Formulierungen des Briefes zeigen zunächst, dass Beale dem Hauptsekretär regelmäßig Berichte geschickt hatte und dass Beale in Cecil einen Patron von höherer Bedeutung sah - und zwar wesentlich höher als seinen einstigen „Ersatzvater“ Hales (der 1565 wegen des Hertford-Grey-Fiaskos inhaftiert worden war). ${ }^{46}$ Beale kommunizierte dies direkt und schrieb an Cecil: „I haue none other frend but you“. ${ }^{47}$ Beales Kommentare über den Kontakt des englischen Botschafters mit den Franzosen zeigen, dass er in vielerlei Hinsicht durch die Beobachtung der Praxis lernte, was ein Diplomat zu tun hatte und was nicht, was in der internationalen Diplomatie funktionierte und was keine Wirkung zeigte. So

42 Vgl. Anm. 27.

43 Taviner, Robert Beale, S. 64, Anm. 48; Nicollier-de Weck, Hubert Languet, S. 85-209.

44 Vgl. z.B. Smiths Dokumente in Beales eigenem Archiv: BL, Additional MSS 48023, 48026, 48047, sowie Smith an Cecil, 09.03.1572, TNA, SP 70/146, fol. 55r.

45 Beale an Cecil, Nov. 1566, TNA, SP 70/87, fol. 75r-76v.

46 Levine, The Early Elizabethan Succession Question, S. 76.

47 Ebd., fol. 76r. 


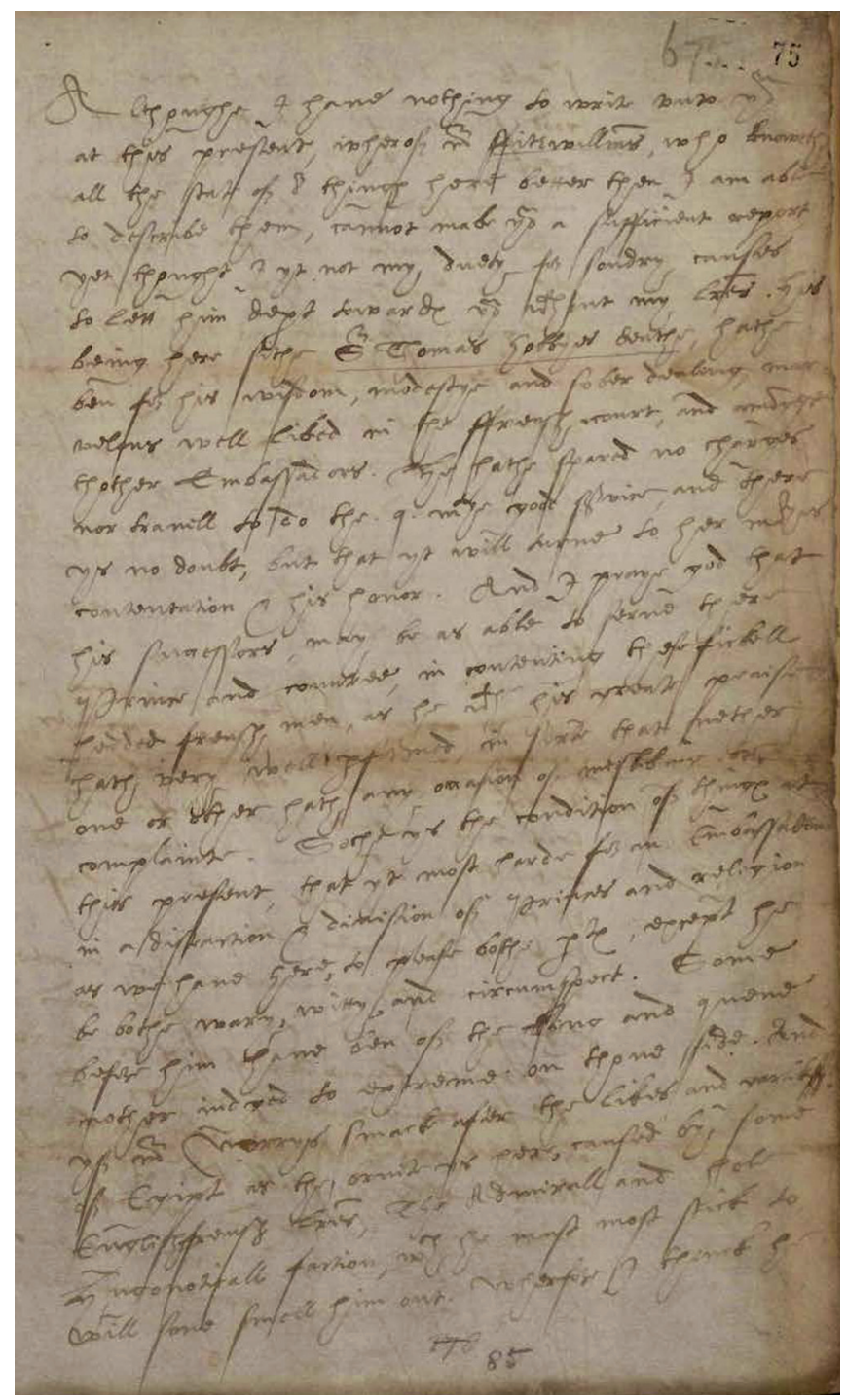

Abb. 5: Robert Beales frühester erhaltener Brief an John Hales and William Cecil

bemerkte er zum Beispiel über die Situation in Paris, dass es sehr schwierig sei, „these fickell hedded frensh men“ in religiösen Angelegenheiten zufriedenzustellen, wenn sich ihre Religion von der des Fürsten unterschied, und der Botschafter „bothe wary, witty, and circumspect“ sein musste. Tatsächlich dachte Beale, dass dies selbst dem ,wisest and politickest man in England not to[o] 
sufficient“ sein konnte. ${ }^{48}$ Allgemeiner schrieb Beale, dass „these dangerous tymes require one bothe of greate wisdom learning, and experience“, wegen der klugen und subtilen Art der Männer in der Regierung. Aus Beales Bemerkungen im Jahre 1566 wird klar, dass die Natur der Diplomatie Männer mit tiefem Wissen und Verständnis erforderte; es bedurfte eines Spezialisten für das Land, in das er entsandt wurde. Für einen Engländer der damaligen Zeit ist dieses Denken sehr vorausschauend, und es deutet darauf hin, dass Beale bereits sehr gut über die Praktiken der italienischen Diplomatie informiert war. ${ }^{49}$

Beale lernte die Umrisse des elisabethanischen diplomatischen Dienstes in Paris während der ersten Jahre der französischen Religionskriege kennen, behielt aber ein nachhaltiges und bald auch nützliches Interesse an den deutschen Angelegenheiten bei, besonders als die Spannungen in den spanischen Niederlanden wuchsen. Im Januar 1568 schickte Beale zum Beispiel zusammen mit einer detaillierten Darstellung der religiösen und politischen Streitpunkte unter den protestantischen Fürsten des Alten Reiches nach London. ${ }^{50}$ Beale beschrieb die beachtlichen militärischen Fähigkeiten des Kurfürsten August von Sachsen (1526-1586) und den Ruf des Kurfürsten von der Pfalz, Friedrich III. (1515-1576), als „Arbiter of the hole cau[s]e“ in Frankreich. Darüber hinaus berichtete er, dass Herzog Christoph von Württemberg (1515-1568) regelmäßig andere Fürsten über seine Korrespondenz mit dem Herzog von Alva (1507-1582) informierte, insbesondere wenn eine Gefahr für die Protestanten wahrscheinlich wurde. Beale lieferte des Weiteren Informationen über die Aktivitäten und Glaubensrichtungen anderer lutherischer Fürsten in Sachsen, der Pfalz, Zweibrücken, Holstein und Braunschweig. Aber sein Fokus blieb stets auf die Wahrscheinlichkeit gerichtet, dass diese deutschen Fürsten die Protestanten in Frankreich unterstützen könnten und würden - trotz der politischen Rivalitäten und religiösen Differenzen untereinander. Diese Beobachtungen waren scharfsinnig. August von Sachsen und Friedrich III. von der Pfalz waren die mächtigsten Protestanten in Deutschland, und Friedrich war fraglos am meisten geneigt, seine konfessionellen Brüder in der Fremde zu unterstützen. Die anderen lutherischen Fürsten schwankten

48 Ebd., fol. 75r, 76r.

49 Ebd., fol. 75v; über Beale und die italienischen relazioni vgl. David Scott Gehring (Hrsg.): Diplomatic Intelligence on the Holy Roman Empire and Denmark during the Reigns of Elizabeth I and James VI. Three Treatises. Cambridge 2016, S. 37.

50 Vgl. TNA, SP 70/96, fol. 65r-66v. 
dagegen. Einige begrüßten zwar militärische Aktionen für religiöse Zwecke, mochten sich darauf aber nicht festlegen lassen. ${ }^{51}$

Beale beschrieb, wie schwierig es war, die verschiedenen deutschen Fürsten für ein Ziel zusammenzubringen, das über die Interessen ihres eigenen Territoriums und ihrer Dynastie hinausging. Letztlich blieb er aber fest davon überzeugt, für die Idee der „protestantischen Internationale“ zu arbeiten. Mit seinen eigenen Worten drückte er es so aus: „As the papists haue theiyr ligue, so were yt not amiss that the princes protestants for theyr defence had the like." ${ }^{25}$

Innerhalb eines Jahres rückten die europäischen Religionskriege bedrohlich an die Niederlande heran, was dazu führte, dass sich die Protestanten in ganz Europa enger zusammenschlossen. Als der pfälzische Kurfürst 1568 schließlich vorschlug, einen Bund zur Verteidigung (der Religion) festzuschreiben, antwortete die englische Königin mit der Entsendung von Henry Killigrew. Es war die bisher ehrgeizigste englische Gesandtschaft an die deutschen Fürsten..$^{53}$

Beale war formal nicht in Killigrews Mission involviert, ähnlich wie er in den 1560er Jahren nie offiziell der englischen Botschaft in Paris angehört hatte. Allerdings hielt er sich in Deutschland auf - und das mit Sicherheit zur richtigen Zeit. Er war in Heidelberg, als Killigrew in Verhandlungen mit dem Kurfürsten von der Pfalz eintrat, und blieb im Umkreis des Kurfürsten, nachdem Killigrew nach England zurückgekehrt war. ${ }^{54}$ Beale erneuerte und schloss Kontakte mit neuen und gleichgesinnten Freunden, wie Hubert Languet und Christoph Ehem. ${ }^{55}$ Währenddessen sammelte er so viel Wissen und Informationen wie möglich über das Heilige Römische Reich im Allgemeinen und über religiöse Angelegenheiten im Besonderen. ${ }^{56}$ Beale reiste mit Killigrew von Heidelberg nach Sachsen und

51 Vgl. Jonas A. M. van Tol: Germany and the French Wars of Religion, 1560-1572. Leiden 2018. Maximilian Lanzinner: Friedenssicherung und politische Einheit des Reiches unter Kaiser Maimilian II. (1564-1576). Göttingen 1993, bes. S. 195-209.

52 TNA, SP 70/96, fol. 66v.

53 Vgl. David Scott Gehring: Anglo-German Relations and the Protestant Cause. Elizabethan Foreign Policy and Pan-Protestantism. London 2013, S. 44-47, 175-177; ders. (Hrsg.), Diplomatic Intelligence, S. $10-16$.

54 De Lumbres an Beale, 11.02.1569, AUL MS 1009/1, Artikel 22; De Lumbres an Beale, 17.04.1569, Brigham Young University, Provo, Utah, MS 457, Artikel 1; Kurfürst Friedrich an Elizabeth, 28.09. 1569, Bodleian Library, Oxford, Ashmole MS 1729, fol. 126r-7v.

55 Für die Korrespondenz von Languet mit Beale vgl. BL, Egerton MS 1693; Ehem an Beale, 06.11. 1570, 16.02.1571, AUL, MS 1009/2, Artikel 27, 28.

56 Vgl. z. B. Languet an Beale, 21.05.1569, BL, Egerton MS 1693, fol. 3r-v; Ehem an Beale, 06.11. 1570, AUL 1009/2, Artikel 27; andere Freunde und Mitarbeiter waren Louis Frennes, Seigneur de Lumbres; Odet de Coligny, Kardinal von Châtillon; und Guillaume Stuart de Vézines; über diese Verbindungen wird die in Vorbereitung befindliche Biographie des Verfassers über Beale detailliert Auskunft geben. 
konnte die Aktivitäten in den kurfürstlichen Residenzen beobachten - wie dort agiert wurde und wie sich die Kurfürsten zu verschiedenen Themen äußerten. Und er studierte die Quellen der Macht und des Reichtums der Kurfürsten. Im Fall von Sachsen stieg Beale sogar in ein Bergwerk ein, um die dortigen hochentwickelten Bergbautechniken zu inspizieren. ${ }^{57}$

In einem Erfahrungsbericht - überschrieben mit dem Titel „The State of Germany“ - fasste Beale schließlich seine Erkenntnisse zusammen, beschrieb die Einnahmen und Kräfte der zehn Reichskreise, die Form und den Ablauf des deutschen Reichstages sowie die Rolle und Vielfalt der Freien Reichsstädte. Er listete zudem in einem Register auf, was es kosten würde, 6000 Reiter und zwei Regimenter Soldaten für ein ganzes Jahr zu unterhalten. ${ }^{58}$ Die Abhandlung enthielt zudem genaue Beschreibungen des Kurfürsten von der Pfalz und des Kurfürsten von Sachsen sowie der jeweiligen Höfe. Beide waren die entscheidenden Akteure, um andere lutherische Fürsten zu bewegen, einem protestantischen Bündnis beizutreten. Beales Beobachtungen über die religiöse Politik des Kurpfälzers und die finanziellen Ressourcen des Kursachsens waren völlig korrekt. ${ }^{59}$ Neben der Beschreibung der politischen Ordnung des Alten Reiches und den Charakterbewertungen zeigte sich Beale hervorragend über die religiösen Überzeugungen der Fürsten informiert. Das wird insbesondere an seiner Darstellung der theologischen Entzweiung der Gnesio-Lutheraner und der Philippisten über Soteriologie und Liturgie deutlich, ebenso wie in seiner Einschätzung, dass genau diese Differenzen die englischen Pläne für ein vereintes, pan-protestantisches Bündnis unmöglich machen würden. Bemerkenswerterweise benannte Beale Matthias Flacius und Georg Maior als jeweilige Repräsentanten der beiden theologischen Lager. ${ }^{60}$ Beales Abhandlung, die sich eng an venezianische relazioni anlehnte, wurde direkt für den Hauptsekretär William Cecil geschrieben. In Anbetracht der früheren Berichte Beales an Cecil liegt es nahe, dass er diesen Bericht über Deutschland Cecil sogar persönlich in London übergab. Er nutzte dazu wahrscheinlich die Gelegenheit, als er - zwar noch nicht als Diplomat, wohl aber als Kurier vom Festland - einen eigenhändig verfassten Brief des pfälzischen Kurfürsten an Elisabeth I. überreichte. ${ }^{61}$

57 Gehring (Hrsg.), Diplomatic Intelligence, S. $72 \mathrm{f}$.

58 Der Bericht liegt ediert vor in ebd., S. 51-108.

59 Vgl. Z. B. Uwe Schirmer: Kursächsische Staatsfinanzen (1456-1656) Strukturen - Verfassung Funktionseliten. Stuttgart 2006; Bernard Vogler: Le rôle des Électeurs palatins dans les Guerres de Religion en France (1559-1592), in: Cahiers d'histoire 10 (1965), S. 51-85

60 Ebd., S. $104 \mathrm{f}$.

61 Ebd., S. 14f., Anm. 35; Christoph Ehem war für Beales Ernennung zum Kurier Königin Elisabeths verantwortlich; vgl. folgende Schreiben: Rat an Friedrich, 28.09.1569, sowie Memoran- 
Um 1570 begann sodann Beales Karriereaufstieg, wobei seine Arbeit für Cecil in Frankreich und in Deutschland förderlich wirkte. Innerhalb eines Jahres trat er offiziell in den Dienst des englischen Diplomaten in der Pariser Botschaft, Francis Walsingham. Im Sommer 1572 avancierte Beale zum Sekretär des Geheimen Rates. Seit diesem Zeitpunkt betrachteten viele im elisabethanischen Regime Beale als einen der führenden Experten für französische und deutsche Angelegenheiten sowie für rechtliche Fragen, und sie taten dies zu Recht. Frühneuzeithistoriker wie Patrick Collinson und einige andere seiner Kollegen, die sich intensiver mit Robert Beale beschäftigt haben, wussten bereits um seine religiösen Neigungen, rechtlichen Interessen und besonderen individuellen Kompetenzen, berücksichtigten allerdings nur seinen zweiten Lebensabschnitt seit den 1570er Jahren. ${ }^{62}$ Das Ausmaß und die Bedeutung von Beales früheren Reisen, Kontakten und Erfahrungen auf dem Kontinent waren dagegen bisher unbekannt. Ohne Familie und mit nur wenigen Freunden gelang es Beale nicht nur, zu überleben, sondern sich während seiner Zeit im Ausland von 1553/54 bis 1569 zu einer weltoffenen Persönlichkeit mit besonderen Fähigkeiten, umfangreichem politisch-konfessionellem Wissen und diplomatischem Talent heranzubilden. Obwohl er in dieser Zeit mit ziemlicher Sicherheit (immer wieder) als Kurier aus Paris nach London beziehungsweise nach England zurückkehrte, war nicht England, sondern das kontinentale europäische Ausland sein Zuhause. Das beweist auch eine Textstelle, mit der Beale von John Herbert - einem Kollegen und Freund in Paris - im August 1570 daran erinnert wurde, dass einige seiner Pakete und Koffer noch immer in Paris seien, obwohl Professor Balduin bereits etliche nach Angers gebracht habe, wo dieser mittlerweile einen Lehrstuhl an der (streng katholischen) Universität angenommen habe. ${ }^{63}$

\section{Fazit und Ausblick}

Beales Aufenthalt auf dem europäischen Festland nachzuzeichnen, ist aufschlussreich. Denn die diversen Aufenthaltsorte und das personelle Netzwerk aus Unterstützern, Freunden, Bekannten und Kollegen zeigen, dass und wie ihn dieser bisher noch nicht untersuchte, erste Teil seines Lebens maßgeblich prägte und ihn jenes Wissen lehrte, das ihm später erfolgreich Verhandlungen im Alten Reich ermöglichte. Seine Flucht aus England fiel zusammen mit der Vertreibung

dum von den Fürsten, 26.09.1569, München, Hauptstaatsarchiv, Kasten Schwarz 16682, fol. 462r464v, 509r-510v.

62 Siehe Anm. 1.

63 AUL, MS 1009/2, Artikel 22, fol. 1r. 
aller Fremden, die kein Bürgerrecht besaßen und keinen Handel betrieben. Es war für Beale der Auftakt, um fortan mit einer Vielzahl von Menschen unterschiedlicher religiöser und nationaler Herkunft zusammenzuleben. Im Alter von 12 oder 13 Jahren war er gewissermaßen dazu konditioniert worden, von Leuten verschiedenster (konfessioneller) Couleur zu lernen und mit ihnen zusammenzuarbeiten, wenngleich er keineswegs als ökumenischer Christ gelten kann. Hier lag ein deutlicher Unterschied zwischen Balduin und Beale: Balduin war ein Ireniker, der versuchte, Protestanten und Katholiken im Rahmen der Religionsgespräche von Poissy im Jahre 1561 und in der folgenden Zeit zu versöhnen. Beale hingegen versuchte, Protestanten zu vereinen, um eventuellen katholischen Angriffen widerstehen zu können. Während dieser Zeit (besonders in den 1560er Jahren) begann Beale allerdings, die Welt des religiösen Konflikts in Schwarz-Weiß zu sehen. In seiner Vorstellung stand der protestantischen Körperschaft - dem Corpus Protestantium - die römisch-katholische Kirche mit ihren politischen Manifestationen feindlich gegenüber. Innerhalb der protestantischen Gemeinschaft sah er verschiedene Grautöne, die sich jedoch trotz der unterschiedlichen theologischen oder ekklesiologischen Anschauungen noch unter dem Banner des Antikatholizismus zusammenschließen konnten. Unter Berufung auf den neutestamentlichen Korintherbrief (1. Korinther 12) benutzte er dies als Argument, als er 1577 die protestantischen Fürsten für einen Bündnisschluss $\mathrm{zu}$ gewinnen suchte. ${ }^{64}$ Für Beale war die Unterstützung und Zusammenarbeit Englands mit der internationalen protestantischen Gemeinschaft sowohl im In- als auch im Ausland schlichtweg eine notwendige Angelegenheit. In England behauptete er, dass er sich in religiösen Angelegenheiten fest an das geltende Recht des Landes gehalten habe. Beale interpretierte die elisabethanische Religionsregelung von 1559, also die Bestimmungen zur Reform und Etablierung der englischen Kirche im protestantischen Sinne, klar als ein protestantisches Bollwerk gegen den Katholizismus. ${ }^{65}$ Im Ausland, wo er die Kunst der Diplomatie erlernt hatte, wusste Beale, wie man mit Protestanten verschiedener Richtungen arbeitete, um eine Einheitsfront zu schaffen.

Was lässt sich also über die Entwicklung von Robert Beale als englischer Diplomat und europäischer Intellektueller sagen? Beale war ein Engländer und zugleich europäischer Intellektueller mit dem Wissen und der Sachkenntnis, um genau diese europäische Sicht diplomatisch in die Tat umzusetzen. Seine Er-

64 Gehring, Anglo-German Relations, S. 72.

65 In der 1580er Jahren sagte John Whitgift, Erzbischof von Canterbury, dass Beale puritanische Neuerungen in die elisabethanische Kirche bringen wollte, aber Beale antwortete, indem er die etablierte Kirche bestätigte. Zu Whitgift und Beale siehe Collinson, The Elizabethan Puritan Movement, S. 243-282. 
fahrung erinnert daran, dass Protestantismus wie intellektueller Austausch im 16. Jahrhundert am besten in einem internationalen, diplomatischen Zusammenhang verstanden werden kann. Am Beispiel Robert Beales können drei Sachverhalte der englischen Diplomatie des 16. Jahrhunderts deutlich gemacht werden:

Erstens wurde Beales Protestantismus in einer internationalen Gemeinschaft von Gelehrten und Theologen aus England, Deutschland, der Schweizer Eidgenossenschaft und Frankreich sowie an verschiedenen Orten in diesen Ländern geformt und geprägt. Während der 1550er und 1560er Jahre wuchsen religiöse Spannungen zu großen kriegerischen Feuersbrünsten heran, aber es ist klar, dass Beale die Konflikte an den konfessionellen Grenzen erfolgreich bewältigte. Er war den Ideen strenger Gnesio-Lutheranern wie dem in Jena lehrenden Matthias Flacius ausgesetzt, schien aber eher unter Philippisten wie Franz Balduin in Heidelberg und Hubert Languet in Wittenberg zu Hause zu sein.

Zweitens: Trotz der internationalen Ursprünge von Beales religiösem Denken war seine Treue zu England - sowohl in der Religion als auch in der Politik unerschütterlich. Gleichwohl wollte er, dass England mit den religiösen Glaubensbrüdern und -schwestern auf dem europäischen Festland zusammenstand. Für ihn konnte das protestantische England nicht vom restlichen protestantischen Europa getrennt werden. Diese konfessionelle Offenheit $\mathrm{zu}$ politischen Zwecken ist wichtig.

Drittens: Beale war ein international denkender Engländer, der aufgeschlossen genug war, um gemeinsam mit anderen Protestanten für politische und publizistische Zwecke zu arbeiten. Dennoch besaß er ein sehr genaues Gespür dafür, was für ihn selbst die richtige und was die falsche Religionsauslegung war. Manchmal präsentierte er eine beindruckende Weitsicht und vorausschauende Perspektive, zu anderen Zeiten biss er sich aber an kleinen Details fest oder war einfach nur pedantisch. Er war ein feinsinniger, juristisch denkender Kopf. Es scheint, als ob er jahrelang einen inneren Groll gehegt hat. Nach eigenen Angaben war er ein bisschen nörgelig. ${ }^{66}$

Beale war möglicherweise moderner als viele seiner Zeitgenossen. Seine Weltanschauung war aufgrund seiner frühen Konfrontation mit verschiedenen politischen und religiösen Perspektiven intellektuell aufgeschlossen. Später konzentrierte sich seine Tätigkeit in London stärker auf einen Ort und auf spe-

66 Vgl. BL, Additional MS 48039, fol. 55r; Historiker des 17. Jahrhunderts und später kommentierten Beales (scheinbar) schlechte Laune und bittere Persönlichkeit; vgl. auch z. B. William Camden: Annales Rervm Anglicarvm et Hibernicarvm, Regnante Elizabetha. London 1615, S. 338; Peter Heylyn: Aërius Redivivus: or the History of the Presbyterians. London 1672, S. $264 \mathrm{f}$;; Thomas Fuller: The Church History of Britain [...]. A New Edition. Bd. 3. London 1837, S. 41 f. 
zifische Fragen der englischen Außenpolitik. Seine internationale Erfahrung war der Grund, weshalb er schließlich offiziell als englischer Botschafter für Elisabeth I. verpflichtet wurde. So war er im Dienste, Her Majesty` ein Engländer, fühlte sich aber wahrscheinlich auch in Deutschland und Frankreich nicht fremd. Beale scheint zugleich Engländer und Europäer gewesen zu sein, je nachdem, aus welcher Perspektive man schaut. 
